a sufficiently high concentration of unligated silver adatoms that their incorporation into the lattice occurs at an appreciable rate. It should be noted that even at -0.6 or $-0.7 \mathrm{~V}$ the Raman intensity slowly decays.

Our hypothesis is that metastable adatoms are created during, or perhaps immediately after, an ORC and are trapped by complexation before they can merge into the metal lattice. These surface complexes are the Raman active sites. This picture explains qualitatively why an ORC is required for SERS, the decrease in intensity at negative potentials, and the irreversible quenching at very negative potentials. The dependence of the number of surface complexes on the halide concentration also explains why water spectra have only been observed at high salt concentrations. With water's small Raman cross section and interference from bulk water, a large number of Raman active surface complexes is needed to produce an observable signal. Other factors, e.g., surface topography, also contribute to the SER effect, but an adatom-adsorbate complex seems to be a necessary component in electrochemically generated SERS. Further investigation is required to confirm this hypothesis and to determine whether this mechanism is a general feature of SERS or is operating only in a few specific cases.

As previously noted, the behavior of the $\mathrm{Cu}-\mathrm{X}(\mathrm{X}=\mathrm{Cl}$, $\mathrm{Br}$ ) spectra is generally similar to the corresponding $\mathrm{Ag}$ systems, but there are differences. The replacement of chloride by bromide (see Figures 5 and 6 ) does not result in a spectrum with bands shifted to lower frequency according to the mass ratio of the halide ions. This implies a difference in the structure of surface complexes on copper and silver, which may be related to the different solution chemistry of these metals.

Acknowledgment. We thank G. Borges for technical assistance.

\title{
Semiempirical Quantum-Mechanical Calculation of the Electronic Structure of DNA. Double-Minimum Potential Curves of the Singlet and Triplet Excited Electronic States,

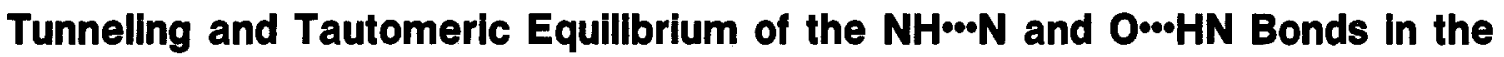 Adenine-Thymine Base Pair
}

\author{
Horaclo Grinberg, ${ }^{\text {1a }}$ \\ Departmento de Quimica Organica, Facultad de Farmacia y Bloquimica, Universidad de Buenos Aires, Buenos Aires, Argentina \\ Alberto L. Capparelli, ${ }^{16}$ \\ Instituto de Investigaciones Fisica-Quimicas Teoricas y Aplicadas (INIFTA), La Plata, Argentina
}

Alejandro Spina, ${ }^{16}$ Julio Marañon, ${ }^{16}$ and Oscar M. Sorarrain ${ }^{\text {1d }}$

Laboratorio de Fisica Teorica, Departamento de Fisica, Facultad de Ciencias Exactas, Universidad Nacional de La Plata, 1900 La Plata, Argentina (Recelved: January 6, 1981; In Final Form: May 1, 1981)

\begin{abstract}
The excited states of the double hydrogen bond in the adenine-thymine nucleotide base pair has been investigated in the semiempirical CNDO/S-CI approximation. Double-minimum potential curves are obtained for several nuclear configurations characterizing simultaneous tautomeric rearrangements of the $\mathrm{NH} \cdots \mathrm{N}$ and $\mathrm{O} \cdots \mathrm{HN}$ bonds. The energy profiles for the coupled movement of the hydrogen bonding show that the Watson-Crick configuration of the adenine-thymine base pair is the most stable for all of the excited states studied. Estimates are made within the WKB approximation of the tunneling rate and tunneling probability. The results indicate that increasing the energy of the excited states would increase the probability of double protonic transfer by tunnel effect and thus for irreversible mutation. A comparison of the composition of the potentials for the single movement of the protons with the double-minimum potential of the concerted movement shows that the potential is nonseparable. The shortcomings that follow from the WKB approximation as applied to the present problem are discussed.
\end{abstract}

\section{Introduction}

The prevalence of hydrogen bonding in chemical and biological systems has led to extensive research into the nature of this interaction by various techniques including molecular orbital methods. In the majority of these studies, the interacting species have been connected by a single hydrogen bond. ${ }^{2}$ There are, however, a number of

(1) (a) Departamento do Química Orgănica, Facultad de Ciencias Exactas y Naturales, Universidad de Buenos Aires, Buenos Aires, Argentina; (b) MCIC CONICET; (c) CONICET fellow; (d) MCIC CIC (Provincia de Buenos Aires, Argentina). biologically important phenomena in which multiple hydrogen bonding plays an integral role; e.g., the base-pair interactions in DNA which are essential to the integrity of the genetic code. ${ }^{3,4}$ In fact, considerable attention devoted to the investigation of tautomerism of nucleic

(2) P. A. Kollman in "Modern Theoretical Chemistry", Vol. 4, H. F. Schaefer, Ed., Plenum Press, New York, 1977, Chapter 3, and references cited therein

(3) J. D. Watson and F. H. C. Crick, Nature (London), 171, 737, 964 (1953); Cold Spring Harbor Symp. Quant. Biol., 18, 123 (1953).

(4) J. S. Kwiatkowski and B. Pullman, Adv. Heterocycl. Chem., 18, 199 (1975). 
bases and their analogues has been partly motivated by the proposeed relationship between the presence of the rare tautomeric form of the base and point mutations that occur during replication., ${ }^{3,6}$ Löwdin, ${ }^{7}$ in effect, suggested that hydrogen-bonded protons can be transferred from one base of DNA to its complement and may play a key role in mutagenesis. These transfers, which could take place either via quantum-mechanical "tunneling" or via more classical means, were proposed to lead to a pair of "rare" tautomeric bases that might be "read" incorrectly during the replication process and result in an altered form of the original DNA molecule.

Early molecular orbital studies of DNA base pairs ${ }^{8-12}$ assumed separability of the $\sigma$ - and $\pi$-electron systems. In this approximation, single proton transfers between the bases were found to be characterized by double-well potentials ${ }^{11}$ as was assumed by Löwdin. More recently, however, an ab initio all-electron calculation of the guanine-cytosine pair ${ }^{13}$ yielded a single-well potential for the transfer of each proton taken one at a time. In an effort to examine multiple simultaneous transfers, the calculations were extended to the study of the hydrogen bonding of the formic acid dimer. Clementi et al ${ }^{13}$ found that a single transfer, which leads to a pair of charged species, is characterized by a monotonically increasing energy function. The double transfer was examined by synchronously coupling the motions of the two protons. This preserves electroneutrality in both species and results in a double-well potential. It was thus concluded that double proton transfers are more likely to produce double-well potentials than are single transfers.

Double-minimum potentials are probably also produced by simultaneous transfer of both protons in singlet and triplet excited states when the nucleic base pairs are exposed to UV electromagnetic radiation. In fact, Lamola et al. ${ }^{14}$ have proposed the phosphorescence of DNA to be a consequence of the double proton transfer in the adenine-thymine base pair. Semiempirical calculations of the double-minimum potential in excited states for the guanine-cytosine pair were performed by Rein et al..$^{10,11 b}$ Later, Blizzard's CNDO/2 calculations seemed to confirm the hypothesis of a double-well potential for the simultaneous transfer of only two protons (upper protons) in the ground state. An extension of the calculations to singlet excited states using the singly occupied virtual orbital approximation seemed to yield no double well. ${ }^{15}$

Since the spectral properties of the bases are chiefly caused by $\pi$ electrons, interpretations of the electronic spectra may be given within the framework of quantummechanical calculations of $\pi$-electronic excitations. Since the middle of the 1960s a number of calculations on the $\pi$-electronic excited states of nucleic bases have been published. ${ }^{4,16}$ Most of these calculations were performed

(5) R. A. Flavell, D. L. Sabo, E. F. Bandle, and C. Weismann, J. Mol. Biol., 89, 255 (1974).

(6) B. Pullman and A. Pullman, Adv. Heterocycl. Chem., 13, 77 (1971).

(7) P. O. Löwdin, Rev. Mod. Phys., 35, 724 (1963); Adv. Quantum Chem., 2, 213 (1965).

(8) B. Pullman and A. Pullman, Biochim. Biophys. Acta, 36, 343 (1959).

(9) H. Bertod and A. Pullman, Biopolymers, 2, 483 (1964); J. Chem. Phys. Phys.-Chim. Biol., 62, 942 (1965).

(10) R. Rein and J. Ladik, J. Chem. Phys., 40, 2466 (1964).

(11) (a) R. Rein and F. E. Harris, Chem. Phys., 41, 3393 (1964); (b) ibid., 42, 2177 (1965); (c) ibid., 43, 4415 (1965); (d) ibid., 45, 1797 (1966) (e) Science, 146, 649 (1964).

(12) S. Lunell and G. Sperber, J. Chem. Phys., 46, 2119 (1967).

(13) E. Clementi, J. Mehl, and W. von Niessen, J. Chem. Phys., 54, 508 (1971).

(14) A. A. Lamola, M. Guéron, T. Yamane, J. Eisinger, and R. G. Shulman, J. Chem. Phys., 47, 2210 (1967).

(15) A. C. Blizzard and D. P. Santry, J. Theor. Biol., 25, 461 (1969) by the CI method using the SCF orbitals, and only singly excited configurations were taken into account. Early studies ${ }^{17}$ including a number of doubly excited configurations seemed to have no significant effect on the calculated spectrum. However, a series of important spectroscopic features points out that the knowledge of $\pi$-electronic wave functions is not sufficient. In this connection the study of the excited singlet and triplet states of the bases with the help of the CNDO/2 method, which permits one to directly take into account the interaction of all valence electrons, has also been undertaken. ${ }^{18,19}$

The controversy between different approximations, which was illustrated in a previous paper ${ }^{20}$ for the ground electronic state study, has become so central to the interpretation of hydrogen bonding in nucleic base pairs that the challenge of studying excited states is irresistible. Therefore, considering the scarcity of theoretical results that enable one to prove the possible existence of the double proton transfer in excited states of the nucleic base pairs, as well as a hypothesis of phosphorescence in DNA, ${ }^{14}$ and taking into consideration previous results related to the tautomerization equilibrium of the isolated bases in excited electronic states, ${ }^{21}$ we have undertaken a systematic study of the coupled proton transfer in both singlet and triplet excited states of the adenine-thymine base pair. Part of this program is reported in the present paper. In order to analyze the possibility of an error mechanism in the genetic code caused by nonradiative processes, such as donor-acceptor interactions between DNA and chemical mutagens, as well as radiation damage, we also include our findings on the tunneling and tautomeric equilibrium of the $\mathrm{NH} \cdots \mathrm{N}$ and $\mathrm{O} \cdots \mathrm{HN}$ bonds in the base pair.

\section{Method of Computation}

All valence electron semiempirical theories are widely used for a variety of purposes. The underlying theory is contained in the early CNDO papers of Pople and coworkers, ${ }^{22}$ but a large number of modifications and new parameterization have emerged ${ }^{23}$ in attempts to develop reliable calculations of ground- and excited-state properties. An important success of CNDO-type theories is that they offer a general scheme for further development. Thus, the natural hierarchy of approximations from CNDO to NDDO and their various modifications such as CNDO/S and MINDO all fall within the original framework.

(16) C. Nagata, A. Imamura, and H. Fujita, Adv. Biophys., 4, 1 (1973).

(17) H. Fujita, A. Imamura, and C. Nagata, Bull. Chem. Soc. Jpn., 41, 2017 (1968).

(18) C. Giessner-Prettre and A. Pullman, Theor. Chim. Acta, 9, 279 (1968).

(19) J. Bertrăn, O. Chalvet, and R. Deudel, An. Fìs. Quim., 66, 247 (1970).

(20) J. Marañön, O. M. Sorarrain, H. Grinberg, S. Lamdan, and C. H. Gaozza, J. Theor. Biol. 74, 11 (1978). See also, S. Abdulnur, "Quantum Science, Methods, and Structure", Plenum Press, New York, 1976, pp $567-75$.

(21) (a) J. Marañôn and O. M. Sorarrain, Z. Naturforsch C, 32, 647 (1977); (b) ibid., 32, 870 (1977).

(22) (a) J. A. Pople, D. P. Santry, and G. A. Segal, J. Chem. Phys., 43, S129 (1965); (b) J. A. Pople and G. A. Segal, ibid., 43, S136 (1965); (c) J. A. Pople, D. L. Beveridge, and P. A. Dobosh, ibid., 47, 2026 (1967); (d) J. A. Pople and D. L. Beveridge, "Approximate Molecular Orbital Theory", McGraw-Hill, New York, 1970.

(23) (a) R. N. Dixon, Mol. Phys., 12, 83 (1967); (b) N. C. Baird and M. J. S. Dewar, J. Chem. Phys., 50, 1262 (1969); (c) J. Del Bene and H. H. Jaffé, ibid., 48, 1807 (1968); (d) D. Fridh, L. Asbrin, and E. Lindholm, Chem. Phys. Lett., 15, 282 (1972); (e) A. Dasgupta and S. Huzinaga, Theor. Chim. Acta, 35, 329 (1974); (f) R. C. Bingham, M. J. S. Dewar and D. H. Lo, J. Am. Chem. Soc., 97, 1285 (1975); (g) N. O. Lipari and C. B. Duke, J. Chem. Phys., 63, 1748 (1975); (h) J. W. H. Kao and A. Chung-Phillips, ibid., 65, 2505 (1976); (i) M. J. S. Dewar and W. Thiel J. Am. Chem. Soc., 99, 4899 (1977); (j) R. Pasternak and A. Y. Meyer, Theor. Chim. Acta, 43, 287 (1977). 


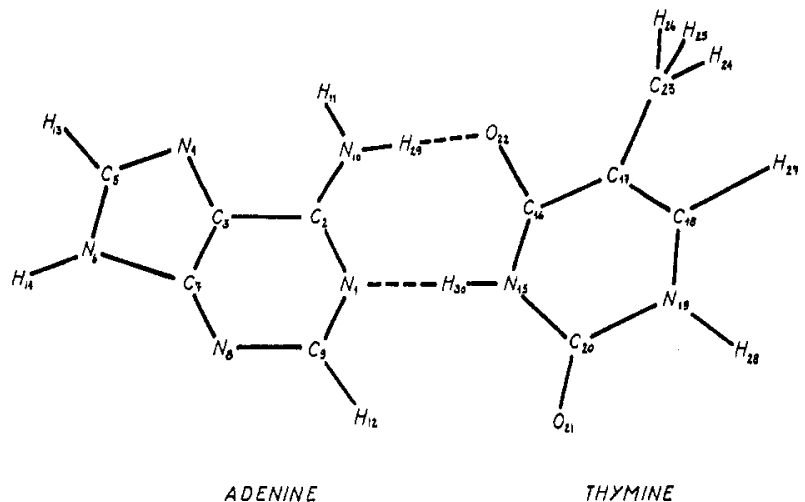

Figure 1. Adenine-thymine base pair. Hydrogen bonds are shown as dotted lines.

For many small molecules, semiempirical theories have been superseded by ab initio calculations either of the STO-NG type or by a more extended CI treatment. However, for moderate- and large-size molecules, these calculations are highly costly and not necessarily more reliable than semiempirical theories. This is particularly true for excited-state properties where ab initio calculations encounter extreme difficulties and where conflicting results have been reported even for small molecules. ${ }^{24}$ In contrast, semiempirical theories have for many years provided reliable spectroscopic calculations that have found widespread applications. It has been found, for example, that $\pi$-electron theories provide a good qualitative understanding of the spectroscopic properties of visual pigments; ${ }^{25}$ however, there are a large number of related problems, such as the description of excited states of the nucleic base pair for which all valence-electron calculations are mandatory.

The failure of the CNDO/2 approximation to correctly reproduce $\sigma-\pi$ separation in planar conjugated molecules seemed to be a particularly severe breakdown in the theory. An incorrect ordering of the $\sigma$ and $\pi$ eigenvectors is obtained, ${ }^{23 \mathrm{c}}$ and higher approximations such as INDO or NDDO are not expected to introduce significant improvement. Yet $\sigma-\pi$ separation seems too fundamental a property to be missed at any level of approximation. Since the CNDO/S-CI method does account for $\sigma-\pi$ separation ${ }^{23 c, 26}$ by removing unnecessary restrictions on the original parameterizations, we have chosen to use this approximation for a description of the singlet and triplet states of the adenine-thymine base pair. It is one of the best methods available at present to study the electronic transitions of large molecules, enabling us to follow the general NDO procedure and making it possible to use a standard Hamiltonian which is applicable to a wide range of problems, minimally including optical spectra, multiplet splittings, oscillator strengths, excited-state charge, and bond-order matrixes; further ground-state information has recently been shown to reproduce closely ab initio calculations. ${ }^{20}$

(24) (a) L. E. McMurchie and E. R. Davidson, J. Chem. Phys, 66, 2959 (1977); (b) R. J. Buenker, S. Shih, and S. D. Peyerimhoff, Chem. Phys. Lett., 44, 385 (1976); (c) R. J. Buenker, S. D. Peyerimhoff and S. Shih J. Chem. Phys., 69, 3882 (1978).

(25) (a) B. Honig, Annu. Rev. Phys. Chem., 29, 31 (1978); (b) B. Honig, A. Greenberg, U. Dimur, and T. Ebrey, Biochemistry, 15, 4593 (1976).

(26) (a) J. Del Bene and H. H. Jaffe, J. Chem. Phys., 48, 4050 (1968); (b) J. Del Bene and H. H. Jaffe, ibid., 50, 1126 (1969); (c) R. L. Ellis, G. Kuehnlenz, and H. H. Jaffe, Theor. Chim. Acta, 26, 131 (1972); (d) K L. Yip, N. O. Lipari, C. B. Duke, B. S. Hudson, and J. Diamond, J. Chem. Phys., 64, 4020 (1976); (e) G. Pouzard and M. Rajzmann, Org. Magn. Reson., 8, 271 (1976); (f) R. W. Bigelow, J. Chem. Phys., 66, 4241 (1977).
The numbering structure of the hydrogen-bonded system under consideration is shown schematically in Figure 1. Bond lengths and angles were obtained from Sutton ${ }^{27}$ and references cited there. The interatomic distances for the atoms involved in the hydrogen bonds were taken from Arnott et al. ${ }^{28}$

Excited-state wave functions were generated from the ground state occupied and virtual orbitals through a configuration interaction procedure between the 30 lowestenergy, singly excited states. ${ }^{29}$ It was felt that inclusion of more Slater determinants would probably not qualitatively alter the present findings. ${ }^{30}$

The problem is some of these studies ${ }^{4,31-33}$ is that, since the exact geometries of the rare tautomeric forms are not known experimentally, the energies were determined by using assumed geometries, which retained the ring structure of the normal form for the rare form.

\section{Results and Discussion}

On theoretical grounds the detailed description of the proton transfer or exchange in hydrogen-bonding systems seems to include two separated steps. The first step is to calculate the potential energy surface for the proton motion. The second problem is to solve the equation of motion for protons on a given potential surface and to evaluate the equilibrium constant or the rate of proton transfer after statistical averaging. In order to describe the first step, one has to define the reaction coordinate, the curve leading from the bottom valley of the initial state over the transition state to the valley of the final state on the potential energy surface inherent to the system concerned. To this end protons $\mathrm{H}_{29}$ and $\mathrm{H}_{30}$ (Figure 1 ) were allowed to move independently along the lines joining $\mathrm{N}_{10}$ and $\mathrm{O}_{22}$ and joining $\mathrm{N}_{1}$ and $\mathrm{N}_{15}$, respectively. All other nuclei were held stationary.

The potential energy curves are shown in Figures 2-5 for both $\pi^{*} \leftarrow \sigma$ and $\pi^{*} \leftarrow \pi$ singlet and triplet excited states as a function of the distance $X$, defined as an orthogonal transformation of the distances of $\mathrm{H}_{29}$ and $\mathrm{H}_{30}$ to $\mathrm{N}_{10}$ and $\mathrm{N}_{15}$, respectively, in the following way:

$$
X=a r_{29}+\left(1-a^{2}\right)^{1 / 2} r_{30}
$$

An isoenergetic scheme of the path followed by the coupled movement of the protons involved in the double hydrogen bonding as given by eq 1 is shown in Figure 6 .

In the adenine-thymine base pair there are 10 carbon atoms, 7 nitrogen atoms, 2 oxygen atoms, and 11 hydrogen atoms. These add up to 98 valence electrons, 76 in socalled $\sigma$ orbitals and 22 in so-called $\pi$ orbitals. (More correctly, neglecting the methyl group on thymine, the adenine-thymine pair is taken as a planar molecule with reflection symmetry in the molecular plane; therefore, the molecular orbitals are of $\mathrm{A}$ and $\mathrm{B}$ type; the 38 doubly occupied A orbitals are those commonly labeled as $\sigma$ orbitals; the $11 \mathrm{~B}$ orbitals are those commonly labeled $\pi$ orbitals. $)^{34}$

(27) L. E. Sutton, Chem. Soc., Spec. Publ., 11, 1958; 18, 1965.

(28) S. Arnott, S. D. Dover, and A. J. Wonacott, Acta Crystallogr. Sect. B, 25, 2192 (1969).

(29) Calculations were performed on an IBM 370/50. The closed-shell CNDO/S-CI program was obtained from the Quantum Chemistry Program Exchange, Indiana University, Bloomington, IN 47401.

(30) J. W. Downing, J. Michl, P. Jorgensen, and E. W. Thulstrup, Theor. Chim. Acta, 32, 203 (1974).

(31) R. Rein, Int. J. Quantum Chem., Quantum Biol. Symp., No. 4, 341 (1971).

(32) R. Rein and R. Garduno in "Quantum Sciences: Methods and Structure", J. Lindenberg et al., Eds., Plenum Press, New York, 1976, p $549 \mathrm{ff}$.

(33) H. Fujita, A. Imamura, and C. Nagata, Bull. Chem. Soc. Jpn., 42, 1467 (1969). 


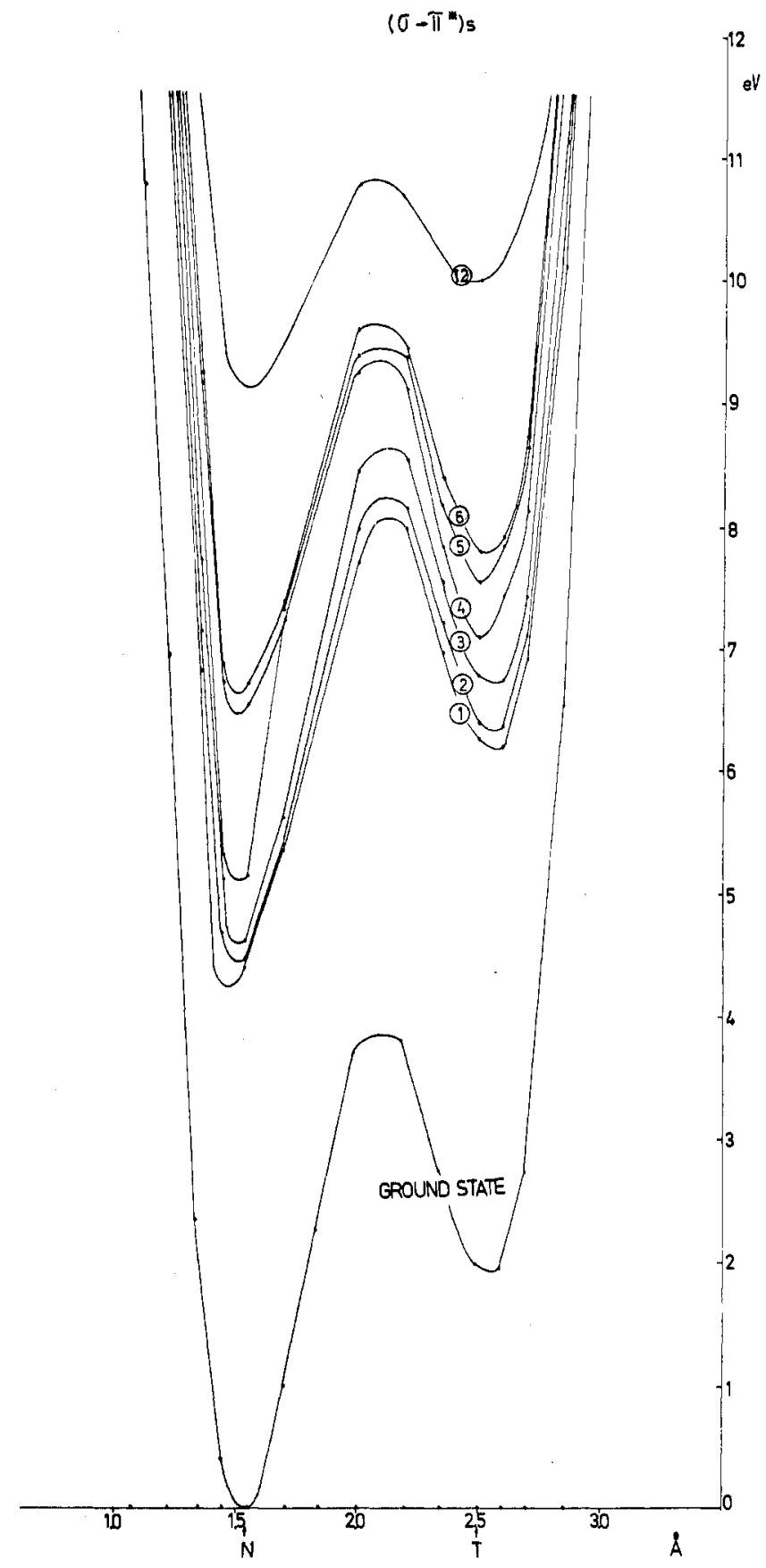

Figure 2. Energy proflles of the $\pi^{*} \leftarrow \sigma$ singlet transitions for the coupled movement of both protons of hydrogen bonds for the adenine-thymine base pair.

Unfortunately, no experimental technique will, at present, allow the detection of a rare tautomeric form present in proportions as low as $10^{-4}$. In principle, this can be solved by means of theoretical calculations of the electronic transitions along the reaction coordinate. Although these studies may not be relevant to tautomeric equilibria in biological systems, they may lead to a better understanding of biological processes where tautomerism is supposed to be involved. Naturally, conclusions obtained in such a way cannot be quantitative; they are heuristic in their character and they allow us to come to

(34) Because the hydrogens of the methyl group of thymine lie out of the plane of the remainder of the molecule, the MOs of $\mathrm{T}$ (and A-T) cannot be strictly identified as pure $\sigma$ or $\pi \mathrm{MOs}$. However, such a classification is possible for most of the relevant MOs as they are of predominantly $\sigma$ or $\pi$ type.

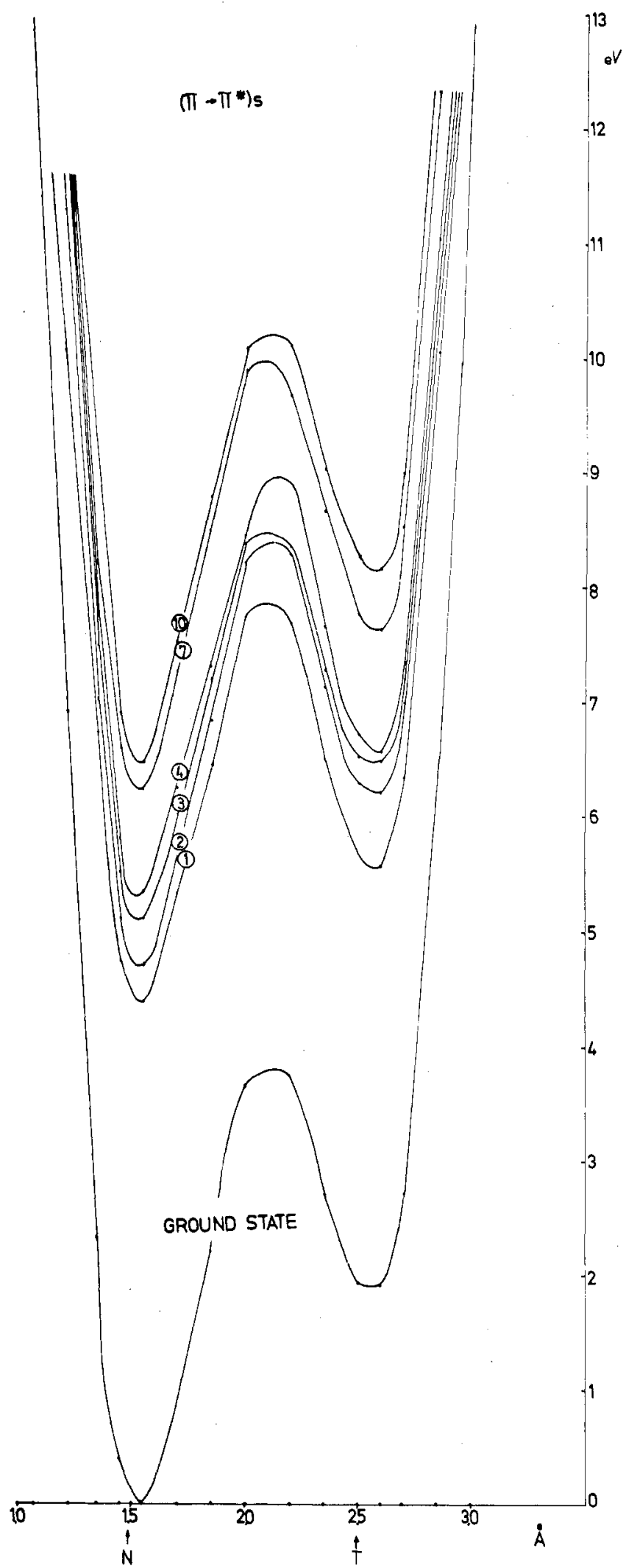

Figure 3. Energy profiles of the $\pi^{*} \leftarrow \pi$ singlet transitions for the coupled movement of both protons of hydrogen bonds for the adenine-thymine base pair.

definite qualitative conclusions about the nature of the hydrogen bond in excited states of the adenine-thymine base pair.

Analysis of Singlet and Triplet Excited States. The $\pi^{*} \leftarrow \pi$ singlet transitions (Figure 3 ) are produced generally between orbitals localized in the same base (A or T); 


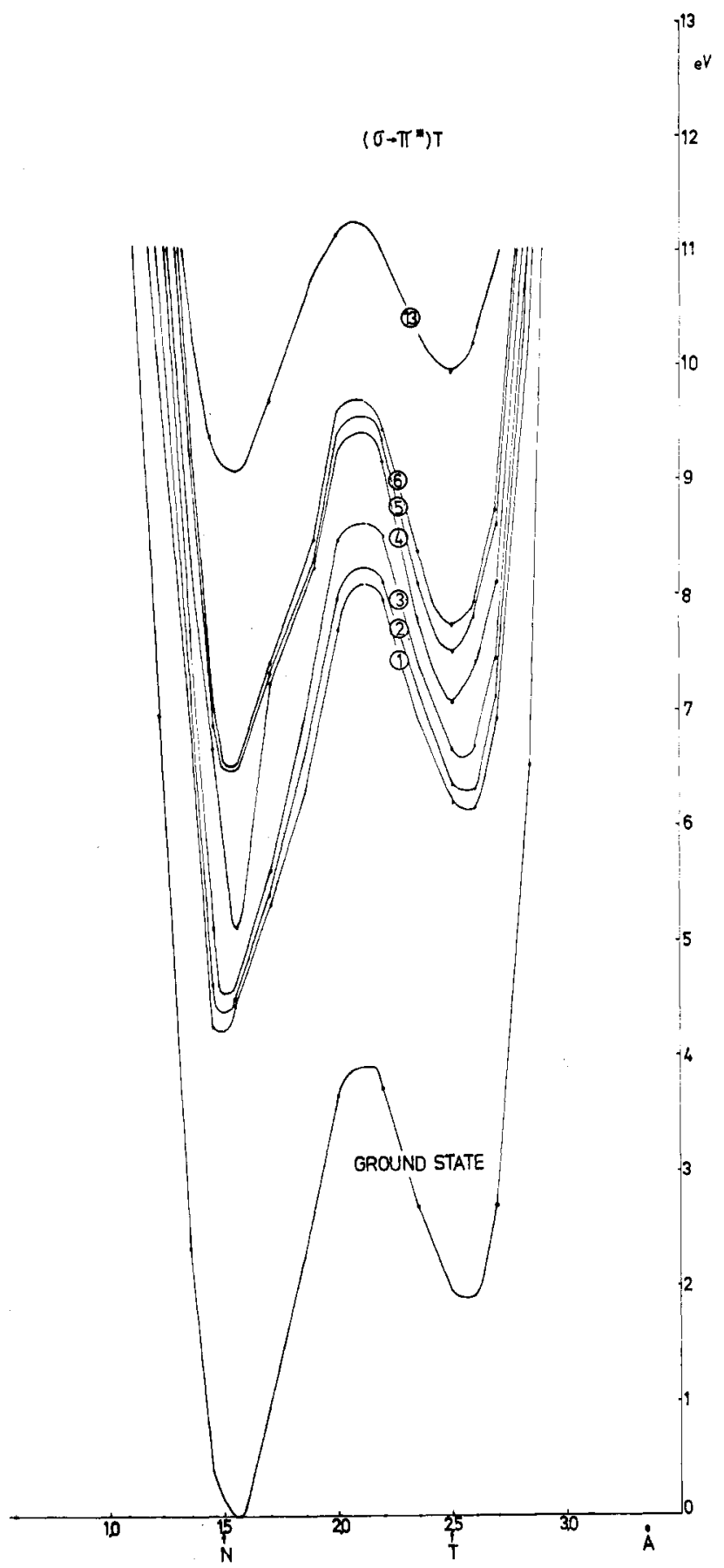

Figure 4. Energy profiles of the $\pi^{*} \leftarrow \sigma$ triplet transitions for the coupled movement of both protons of hydrogen bonds for the adenine-thymine base pair.

i.e., they are intramolecular in nature, except the $4 \pi^{*} \leftarrow$ $\pi$ and $11 \pi^{*} \leftarrow \pi$ transitions of the rare tautomeric form. In fact, the $4 \pi^{*} \leftarrow \pi$ transition is produced exclusively by the promotion of one electron from HOMO (T) to LUMO (A); the $11 \pi^{*} \leftarrow \pi$ transition is due to the electronic promotion from HOMO (A) to LUMO + 4 (T). These transitions are strongly associated with the behavior of the two protons of the hydrogen bonds, $\mathrm{NH} \cdots \mathrm{N}$ and $\mathrm{O} \ldots \mathrm{HN}$, since they are generated by the promotion of electrons localized in mixture orbitals, lone pair and $\pi$ of adenine to localized orbitals in double bonds of thymine $(\mathrm{C}=\mathrm{O}$ and $\mathrm{C}=\mathrm{C}$ bonds). That is, an optical transition between levels originating from different bases would involve a charge transfer between the two bases. The same trend is observed in the $\pi^{*} \leftarrow \pi$ triplet transitions (Figure 5). Here

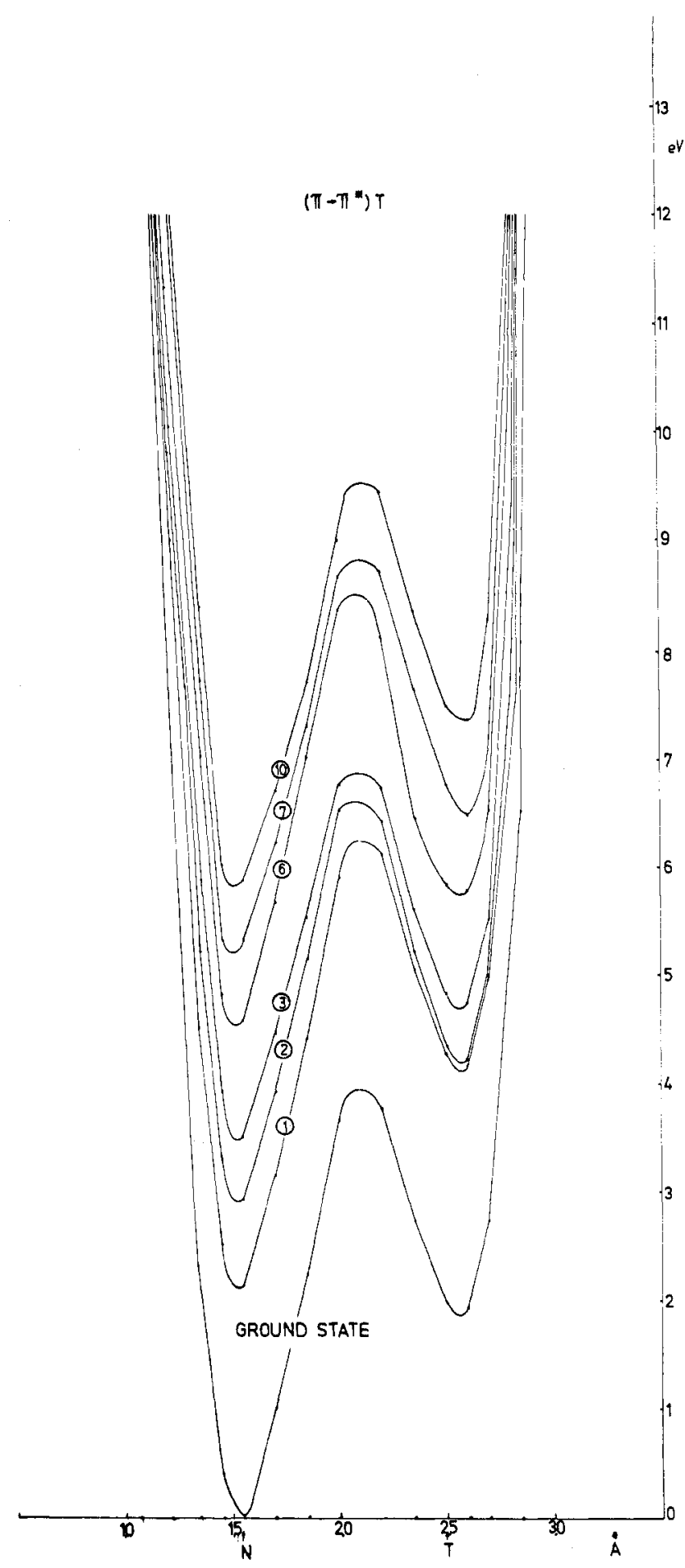

Figure 5. Energy profiles of the $\pi^{*} \leftarrow \pi$ triplet transitions for the coupled movement of both protons of hydrogen bonds for the adenine-thymine base pair.

the lowest energy transfer for the normal form is predicted to be $2.1 \mathrm{eV}$ and that of the tautomeric form is calculated to be $4.1 \mathrm{eV}$. We can anticipate increased stability of the normal form relative to the right.

Similar effects are probably expected if the heavy atoms of the bases are allowed to relax their positions as the proton transfers occur. This alternative, although achievable in principle, would have been an exceedingly laborious procedure and was not considered in the present work. Calculations on the formic acid dimer ${ }^{35}$ in fact

(35) (a) S. Scheiner and C. W. Kern, Chem. Phys. Lett., 57, 331 (1978); (b) S. Scheiner and C. W. Kern, J. Am. Chem. Soc., 101, 4081 (1979). 


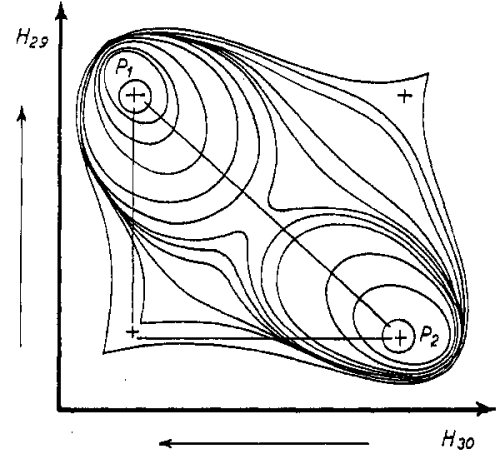

Figure 6. Isoenergetic scheme of the path followed by the coupled movement of the protons involved in the double hydrogen bonding of the adenine-thymine base pair. $P_{1} P_{2}$ is the reaction coordinate generating the double-minimum potential (see eq 1).

suggest that the barriers to double proton transfers may also be facilitated by uncoupling the motions of the protons or by zero-point vibrational deformations. ${ }^{36}$ All of these effects would tend to reduce the CNDO/S-CI barrier in both the ground and excited states.

As far as the $\pi^{*} \leftarrow \sigma$ singlet and triplet transitions (Figures 2 and 4 ) of both normal and tautomeric forms is concerned, they are due to the promotion of electrons delocalized on both bases to orbitals localized in a single base $\pi^{*}(\mathrm{~A}$ or $\mathrm{T}) \leftarrow \sigma(\mathrm{D})$. The delocalized $\sigma$ orbitals have a strong component of the lone-pair electrons belonging to the hydrogen bonds.

Previous ab initio calculations $\mathrm{s}^{35 \mathrm{~b}, 37-40}$ have found the highest occupied and lowest unoccupied molecular orbitals (MOs) of each base to be of $\pi$ symmetry. Our CNDO/S-CI calculations indeed agree with this result; in addition, we find the highest occupied and lowest vacant MOs of the A-T base pair to be of $\pi$ symmetry as well. Clementi et al. ${ }^{13}$ have reached a similar conclusion for the highest occupied MO of the guanine-cytosine base pair. First ionization potentials are thus predicted to be of $\pi$ symmetry, and excitations of lowest energy are of $\pi^{*} \leftarrow \pi$ type. A more specific result of this method concerns the status of the lone pairs. Thus, although atomic "lone pairs" do not exist as such (pure) in molecules, the analysis of the coefficients of the atomic orbitals in the MOs shows very neatly that a large amount of "lone-pair character" may be assigned to the highest $\sigma$ levels in these heterocycles. Although deeper in energy than the highest $\pi$ level, these "lone pairs" (or combined lone pairs) appear more ionizable than the other $\sigma$ electrons, with oxygen lone pairs more ionizable than nitrogen lone pairs.

The characterization of a double-minimum potential, typical of a double transfer, is given by the barrier height $V$ and upper well height $A$. The variation of these parameters as a function of the energy of the normal form for each excited state (singlet and triplet transitions) below $10 \mathrm{eV}$ is given in Figures 7 and 8.

The behavior of the barrier height $V$ (Figure 7) for both the $\pi^{*} \leftarrow \sigma$ and $\pi^{*} \leftarrow \pi$ singlet and triplet transitions is quite different from that shown in Figure 8 for the vari-

(36) W. A. Sokalski, H. Romanowski, and A. Jaworski, Adv. Mol. Relaxation Interact. Processes, 11, 29 (1977).

(37) E. Clementi, J. M. Andre, M. Cl. Andre, D. Klint, and D. Hahn, Acta Phys, Acad. Sci. Hung., 27, 493 (1969).

(38) R. E. Christoffersen and R. P. Angelli in "New World of Quantum Chemistry", B. Pullman and R. Parr, Eds., Reidel, Boston, 1976, pp 189-210.

(39) B. Mely and A. Pullman, Theor. Chim. Acta, 13, 278 (1969).

(40) L. C. Snyder, R. G. Shulman, and D. B. Neumann, J. Chem. Phys., 53, 256 (1970).
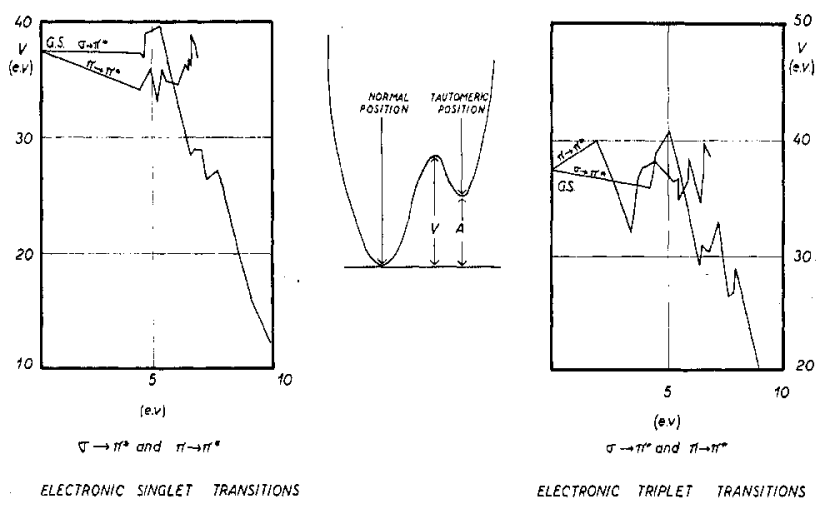

Figure 7. Correlation of barrier height $V$ for $\pi^{*} \leftarrow \sigma$ and $\pi^{*} \leftarrow \pi$ singlet and triplet transitions in the adenine-thymine base pair as a function of the energy of the normal form.
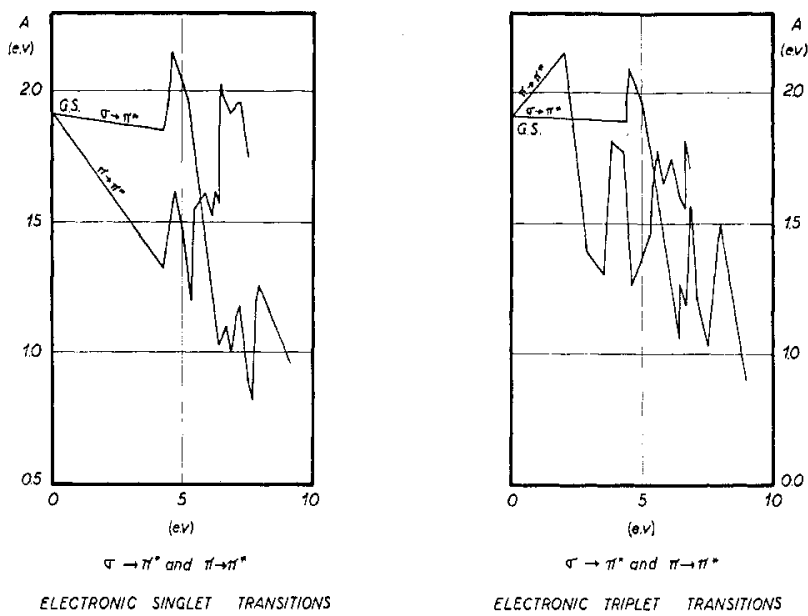

Figure 8. Correlation of upper well helght $A$ for $\pi^{*} \leftarrow \sigma$ and $\pi^{*} \leftarrow$ $\pi$ singlet and triplet transitions in the adenine-thymine base pair as a function of the energy of the normal form.

ation of the upper well height $A$. For the $\pi^{*} \leftarrow \pi$ singlet and triplet transitions it can be observed that $V$ is practically independent of the energy of the transitions. As to the $\pi^{*} \leftarrow \sigma$ transitions (singlets and triplets), they show an abrupt diminution of the height as the excitation energy increases from the $4 \pi^{*} \leftarrow \sigma$ transition, which would indicate that increasing the energy of the excited states would increase the probability of double protonic transfer by tunnel effect. This phenomenon is quantitatively analyzed below.

It can be observed that the variation of the $A$ parameter for the $\pi^{*} \leftarrow \pi$ singlet transitions (Figure 8 ) tends to increase the difference of the mimina from the $1 \pi^{*} \leftarrow \pi$ transition; i.e., they manifest a trend to increase the asymmetry of the potential profiles. As to the $\pi^{*} \leftarrow \pi$ triplet transitions, their behavior is approximately constant; i.e., the asymmetry of the profiles is energy independent. The $\pi^{*} \leftarrow \sigma$ singlet and triplet transitions show a trend to decrease the difference of the minima with the excitation; that is, the profiles tend ot the symmetry with the increase of the excitation energy.

An additional observation which could be mentioned at this point is that the particularly large diminution of $V$ from the $4 \pi^{*} \leftarrow \sigma$ to the $5 \pi^{*} \leftarrow \sigma$ singlet transitions probably marks the perfectly defined separation of two bands of fluorescence. From the analysis of the variation of the barrier height $V$ and the difference $A$ of the two minima for the $\pi^{*} \leftarrow \sigma$ singlet and triplet states as a function of the excitation energy of the normal form, it can be observed that a trend to the symmetry of the potential 
TABLE I: Tunneling and Equilibrium Data for the Singlet Excited States of the A-T Base Pair ${ }^{a}$

\begin{tabular}{rrrrrrr}
\hline state & $s$ & $g$ & $f_{T}, \mathrm{~s}^{-1}$ & $f_{\mathrm{N}}, \mathrm{s}^{-1}$ & $\tau, \mathrm{s}$ \\
\hline $1\left(\pi^{*} \leftarrow \pi\right)$ & 16.57 & $4.05 \times 10^{-15}$ & $1.22 \times 10^{12}$ & $0.86 \times 10^{12}$ & $1.18 \times 10^{2}$ & $4.11 \times 10^{-18}$ \\
$10\left(\pi^{*} \leftarrow \pi\right)$ & 15.86 & $1.67 \times 10^{-14}$ & $1.27 \times 10^{12}$ & $0.86 \times 10^{12}$ & $2.81 \times 10^{1}$ & $6.57 \times 10^{-27}$ \\
$1\left(\pi^{*} \leftarrow \sigma\right)$ & 14.42 & $2.98 \times 10^{-13}$ & $2.16 \times 10^{12}$ & $1.04 \times 10^{12}$ & $1.05 \times 10^{0}$ & $9.50 \times 10^{-33}$ \\
$12\left(\pi^{*} \leftarrow \sigma\right)$ & 8.91 & $1.82 \times 10^{-8}$ & $2.57 \times 10^{12}$ & $1.38 \times 10^{12}$ & $1.39 \times 10^{-5}$ & $5.56 \times 10^{-15}$
\end{tabular}

${ }^{a}$ See ref 20 for the explicit meaning of the tunneling and equilibrium data. ${ }^{b}$ Equilibrium constant computed at $T=$ $310 \mathrm{~K}$.

TABLE II: Tunneling and Equilibrium Data for the Triplet Excited States of the A-T Base Pair ${ }^{a}$

\begin{tabular}{rrrrrrr}
\hline state & $s$ & $g$ & $f_{\mathrm{T}}, \mathrm{s}^{-1}$ & $f_{\mathrm{N}}, \mathrm{s}^{-1}$ & \multicolumn{1}{c}{$\mathrm{s}$} & $K^{b}$ \\
\hline $1\left(\pi^{*} \leftarrow \pi\right)$ & 12.49 & $1.42 \times 10^{-11}$ & $1.84 \times 10^{12}$ & $1.04 \times 10^{12}$ & $2.44 \times 10^{-2}$ & $1.72 \times 10^{-33}$ \\
$10\left(\pi^{*} \leftarrow \pi\right)$ & 11.65 & $7.60 \times 10^{-11}$ & $1.94 \times 10^{12}$ & $1.08 \times 10^{12}$ & $4.36 \times 10^{-3}$ & $5.72 \times 10^{-28}$ \\
$1\left(\pi^{*} \leftarrow \sigma\right)$ & 11.05 & $2.51 \times 10^{-10}$ & $3.11 \times 10^{12}$ & $0.96 \times 10^{12}$ & $9.62 \times 10^{-4}$ & $1.97 \times 10^{-33}$ \\
$13\left(\pi^{*} \leftarrow \sigma\right)$ & 8.50 & $4.13 \times 10^{-8}$ & $1.94 \times 10^{12}$ & $1.22 \times 10^{12}$ & $7.66 \times 10^{-6}$ & $2.25 \times 10^{-16}$
\end{tabular}

${ }^{a}$ See ref 20 for the explicit meaning of the tunneling and equilibrium data. ${ }^{b}$ Equilibrium constant computed at $T=$ $310 \mathrm{~K}$.

profiles at energies below the ionization energy of the orbitals is involved in the transitions. The above-mentioned behavior of the singlet transitions is also observed for the triplet states. As suggested, ${ }^{14}$ the two-proton transfer in the A-T pair under irradiation can also be a cause of the phosphorescence band of DNA. Realization of such a possibility would mean that in the triplet state the two-proton mechanism takes place. ${ }^{41}$

Equilibria and Tunneling. Although most experimental genetic systems do not require quantum theory for interpretations, ${ }^{42}$ the experiments of Freese and Freese ${ }^{43}$ and Kornberg ${ }^{44}$ indicate that the high fidelity of DNA replication is due to an extremely sensitive hydrogen-bonddirected base-pairing process where any misincorporations are enzymatically connected afterward by "copy-editing" enzymes. ${ }^{45,46}$ These enzymes would apparently discriminate against rearranged proton-electron configurations of the template strand which would therefore decrease the probability of observing most biological consequences generally associated with the replication of unusual tautomeric bases. Experiments designed to investigate the microscopic model ${ }^{7}$ of genetic information must therefore take into account the possibility of enzymatic masking of proton-electron arrangements which could have occurred before replication. Other experimental difficulties are exemplified by the fact that experimental investigation of proton tunneling reactions ${ }^{47}$ in well-defined chemical systems was delayed $20 \mathrm{yr}$ after Bell ${ }^{48}$ formulated the process in terms of basic theoretical and experimental principles. This delay was not due to an absence of proton tunneling reactions but to an absence of "proper" experimental methods. ${ }^{49}$ Efforts to detect proton tunneling in a complicated ill-defined in vivo environment should likewise eliminate all possible "experimental difficulties" associated with detection. Thus, it is not surprising that direct experimental investigation of the microscopic model of genetic information is scarce; ${ }^{50}$ nevertheless, theoretical

(41) V. I. Danilov, V. I. Pechenaya, and N. V. Zheltovsky, Int. J. Quantum Chem., 17, 307 (1980).

(42) W. G. Cooper, Int. J. Quantum Chem., Quantum Biol. Symp., No. 5, 463 (1978).

(43) E. B. Freese and E. Freese, Proc. Natl, Acad. Sci. U.S.A., 57, 650 (1967).

(44) A. Kornberg, Science, 163, 1410 (1969); “DNA Synthesis", W. H. Freeman, San Francisco, 1974.

(45) J. J. Hopfield, Proc. Natl. Acad. Sci. U.S.A., 71, 4135 (1974). (46) J. D. Watson, "Molecular Biology of the Gene", 3rd ed., W. A. Benjamin, Reading, MA, 1976.

(47) R. P. Bell, J. A. Fendley, and J. R. Hulett, Proc. R. Soc. London, Ser. A, 235, 53 (1956).

(48) R. P. Bell, Proc. R. Soc. London, Ser. A, 148, 241 (1935).

(49) E. F. Caldin, Chem. Rev., 69, 135 (1969). investigations ${ }^{36}$ continue to imply that quantum-mechanical properties of genetic information may be required to explain particular biological observations. However, the fact that the immediate environment of an in vivo molecular genetic system is essentially unknown severely restricts the information contained in most atomic and molecular model calculations. Thus any real qualitative success with such models is considered significant. Therefore, we have decided to investigate the tunnel effect in both singlet and triplet excited states of the A-T base pair in both normal and tautomeric forms.

The modes of proton transfer investigated thus far have allowed no motion of the heavy nuclei which were assumed to be stationary. Since the much bulkier atomic framework of the base pair is considerably less mobile than the protons being transferred, this is expected to provide a reasonably good basis for studying quantum-mechanical tunneling.

Rein and Harris ${ }^{11}$ have estimated the equilibrium proportions of the tautomers formed as a result of the transfer of upper protons in the guanine-cytosine pair. They have also computed the times required for each of these protons to tunnel to the complementary base. Their results were based on semiempirical molecular orbital calculations on (primarily) the $\pi$-electron system of the base pair which yielded double-well potentials for single proton transfers, contrary to the all-electron calculations reported by Clementi et al. ${ }^{13}$ and by Scheiner et al. $^{35}$ in the ground state. Since our all-valence electron calculations of the double transfer in the excited states do, by contrast, yield double-well potentials, we have computed values for the equilibrium constant as well as the tunneling time and the classical oscillatory frequency associated with these modes.

The application of kinetic and quantum-mechanical principles to tunneling and equilibria in double-well potentials has been summarized in a convenient form by Löwdin. ${ }^{7}$ The probability of an incident particle tunneling through a barrier is, in the WKB approximation, dependent upon the quantity

$$
s=\hbar^{-1} \int_{z_{1}}^{z_{2}}\{2 m[V(z)-E]\}^{1 / 2} \mathrm{~d} z
$$

where $m$ and $E$ are the mass and the energy of the particle, $V(z)$ is the potential energy in each excited state at position $z$, and $z_{1}$ and $z_{2}$ are the tunneling points, i.e., the limits of the classically forbidden portion of the path $\left(V(z) \geq E, z_{1}\right.$ $\leq z \leq z_{2}$ ). The tunneling and equilibrium data for both the singlet and triplet excited states of the $\mathrm{A}-\mathrm{T}$ base pair

(50) J. W. Drake and R. H. Baltz, Annu. Rev. Biochem., 45, 11 (1976). 


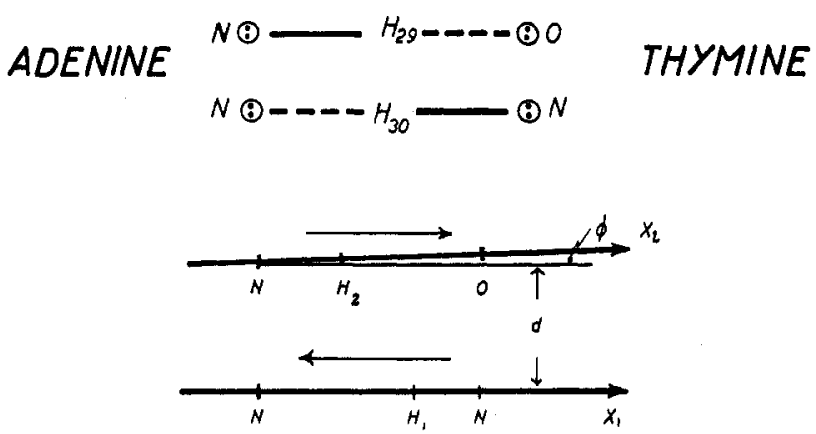

Figure 9. Mechanical model of the double hydrogen bonding of the adenine-thymine base pair.

are presented in Tables I and II. Details of the meaning of and the assumptions made in the explicit calculation of the barrier parameter (eq 2), the tunneling probability $g$, the left and right oscillation frequencies $f_{\mathrm{N}}$ and $f_{\mathrm{T}}$, the tunneling time $\tau$, and the equilibrium constant $K$ have been described in a previous paper ${ }^{20}$ and will not be repeated here.

In these calculations we have neglected the correction due to zero-point vibrational energy in both minima for each excited state; i.e., it was assumed that the energies can be taken directly from the values corresponding to the minima. Of course, this assumption does not change the character of the values given in Tables I and II.

It is evident that, if the time required by the proton to tunnel through the potential hill in the medium of the hydrogen bonds of the A-T base pair is small as compared to the lifetime of a singlet excited state $\left(10^{-8} \mathrm{~s}\right)$, the conditions will be particularly favorable for the appearance of ionic tautomeric forms and thus for irreversible mutation. In fact, the $13 \pi^{*} \leftarrow \sigma$ singlet and triplet transitions (Tables I and II) show that there exists a finite probability of double proton tunneling tautomerization which could be responsible for the partial loss of the genetic code. This is particularly true for the triplet state, since its lifetime is much longer than that of a typical singlet state.

The present calculation as proposed by Löwdin ${ }^{7}$ is based on the phase-integral approximation, sometimes called the WKB method ${ }^{51}$ for unidimensional physical models, assuming that the barrier penetration is due to the movement of one proton in just one potential well. More generally, the problem which we are dealing with here is that of the double simultaneous displacement of two protons in both hydrogen bondings of the A-T base pair, in which the resulting potential is a two-dimensional double-minimum potential $V\left(x_{1}, x_{2}\right)$ (see Figure 9).

According to the physical model proposed in Figure 9, the Hamiltonian of two particles (two protons) can be written

$$
\mathscr{H}\left(p, x_{1}, x_{2}\right)=p^{2} / m+V\left(x_{1}, x_{2}\right)
$$

where $x_{1}$ and $x_{2}$ are the support lines of the classical trajectories which contribute to the tunneling process. It can be observed that these two lines are not parallel, so that the coordinate of proton 2 can be expressed as a function of an angular parameter $\phi$

$$
x_{2}=x_{1}+d \sin \phi
$$

Therefore, the Hamiltonian takes the form

$$
\begin{aligned}
\mathscr{H}\left(p_{x_{1}}, p_{\phi} ;\right. & \left.x_{1}, \phi\right)= \\
& p_{x_{1}}{ }^{2} / m+\left(p_{x_{1}}+\mathrm{d} \cos \phi p_{\phi}\right)^{2} / m+V\left(x_{1}, \phi\right)
\end{aligned}
$$

(51) See, for example, A. S. Davydov, "Quantum Mechanics", Pergamon Press, Elmsford, NY, 1969.

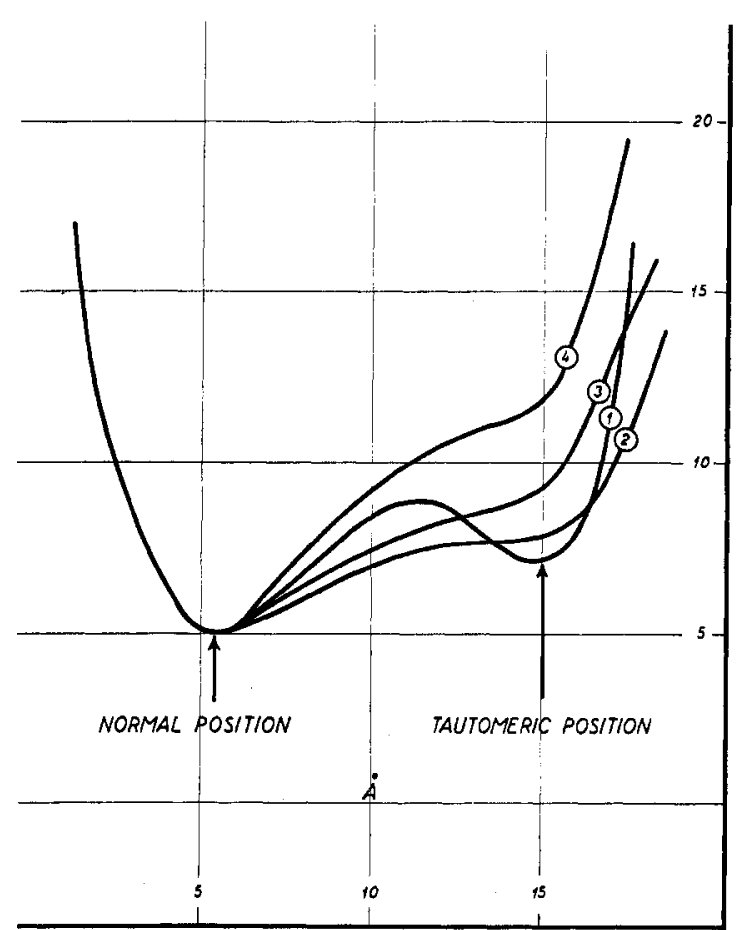

Figure 10. Comparison of the potential curves of the composite movement with the curve resulting from the composition of the single hydrogen bonding $1 \rightarrow 4$ : (1) double-minimum potential (A-T); (2) potential of the hydrogen bond $\mathrm{H}_{30}$; (3) potential of the hydrogen bond $\mathrm{H}_{29}$; (4) sum of the potentials of the hydrogen bonds $\mathrm{H}_{29}$ and $\mathrm{H}_{30}$. All curves refer to the ground state.

Clearly, the WKB method cannot be applied to the two-dimensional potential $V\left(x_{1}, \phi\right)$ since this is a nonseparable potential. This means that the potential cannot be described as a sum of potentials of just one hydrogen bonding $V_{\mathrm{i}}(x)$, i.e.

$$
V\left(x_{1}, \phi\right) \neq V_{1}\left(x_{1}\right)+V_{2}(\phi)
$$

The justification of the nonseparability of the potential $V\left(x_{1}, \phi\right)$ is found in the following facts. From the atomatom bond index curves of the $\mathrm{A}-\mathrm{T}$ base pair for the single displacement, ${ }^{20}$ a net transfer of electronic charge can be observed in the opposite direction to that of the proton's movement. On the other hand, the curves for the coupled motion of the two protons show the protonic and electronic currents to cancel each other, thus making the total current in the ring comprising the two hydrogen bondings and the rest of the system vanish. Therefore, it is not possible to decompose the concerted movement of both protons as the sum of the movements of each of them. A second justification is found by comparing the composition of the potentials for the single movement of the protons with the double-minimum potential of the concerted movement (Figure 10).

It can be clearly seen that the sum of the potentials is not equal to the potential of the concerted movement of both protons. Further, a cooperativity effect seems to operate, since the potential due to the movement of a single proton is determined by the position of the second one in the other potential well. In Figure 10 it is considered that, when a proton is moved along the line constituting the hydrogen bond, the other is in its normal position; i.e., the minima coincide with those of the potential of the coupled motion of both protons; in this case the coincident minimum represents the normal position of the $\mathrm{A}-\mathrm{T}$ base pair. A similar result could, of course, be obtained if the movement of one proton is performed as the second one is in the tautomeric position, giving the same relative 

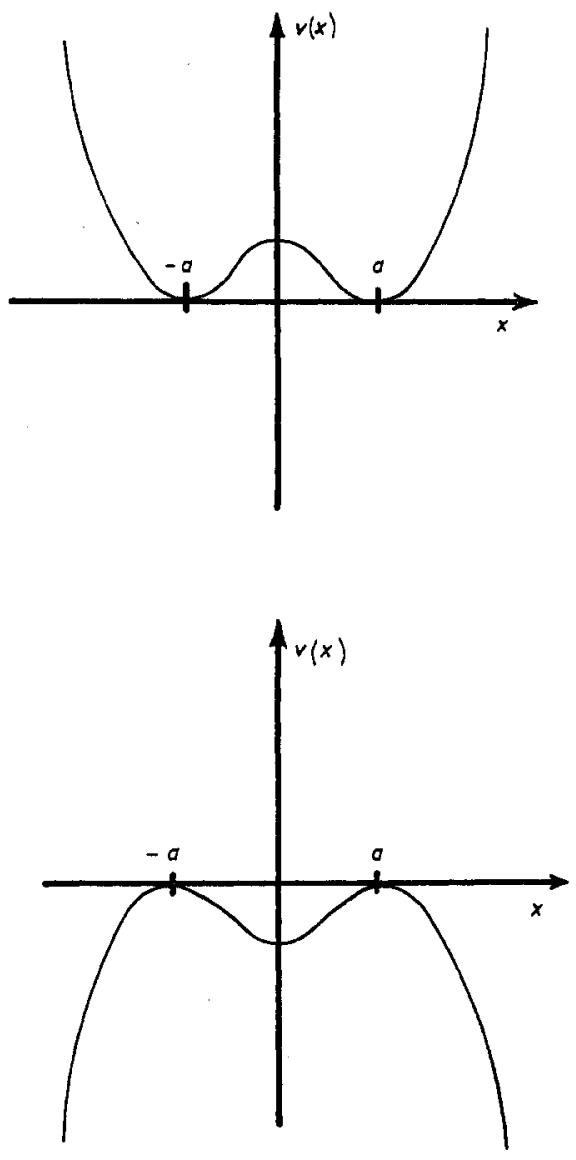

Figure 11. See text.

minimum in the position corresponding to the tautomeric form of the $\mathrm{A}-\mathrm{T}$ base pair. Therefore, in view of these results, one can only infer that the double-minimum potential cannot be decomposed as the sum of the separated potentials for each proton.

\section{Conclusions}

From the analysis made for the energy profiles of the $\pi^{*} \leftarrow \sigma$ singlet and triplet transitions, it can be observed that, for energies close to the ionization, the double-minimum potential curves tend to become symmetrical and the barrier heights become lower. Consequently, the following remarks can be mentioned.

(a) The energy profiles for the coupled movement of the $\mathrm{H}$-bond protons show that, with the excitation of the system, the asymmetry of the potential curves does not change; that is, the transitions of the normal form occur at higher wavelengths than those of the tautomeric form, and therefore, in all of the states studied, the WatsonCrick configuration of the A-T pair is the most stable one. Thus, the theoretical results do not confirm the hypothesis that the red shift of the DNA fluorescence band is due to the two-proton transfer.

(b) The tunneling equilibrium reported in the present work is based on the approximation that the two minima in Figures 2-5 for the different excited states are parabolic. Since anharmonic effects are believed to be relatively small, the parabolic approximation is acceptable. Within this context, there exists the possibility of simultaneous double proton tunneling tautomerization for excitation energies higher than $12 \mathrm{eV}$. This is particularly true for the triplet state (see Table II).

The tautomerization processes must have other origins, i.e., electronic charge transfer derived from donor-acceptor interaction with other molecules or ionization effected by radiation.

The effects just discussed, though important, are probably less significant than the uncertainty in rate introduced by limitations of our knowledge of the barrier shape. Actually, if it should be possible to analytically represent the potential $V(x, \phi)$, the problem of calculating the tunneling process could be carried out by using the different methods that at present are available. Among these can be mentioned the one developed by Gutzwiller ${ }^{52}$ as applied to single-minimum potentials of multidimensional and nonseparable problems. More recently, Coleman ${ }^{53}$ working in Minkowski space and in four-dimensional Euclidean space $^{54}$ has shown that the double-well problem is closely related to the concept of "instantons" or "solitons", what should be the solution of the equation of motion for a classical particle in a symmetrical double-minimum potential as shown in Figure 11. Thus, when a rotation of the time to a Euclidean imaginary time is performed, the potential $V(x)$ is inverted to $-V(x) .^{55}$ On making use of this idea, Chang ${ }^{56}$ studied the tunneling phenomenon involving two degrees of freedom. He found the most probable escape path in phase space (which links the initial and final states) and the classical trajectories between the relative maxima and applied this philosophy to the classical problem of the inversion of ammonia.

Another factor not explicitly considered in the present work is the sensitivity of $f_{\mathrm{N}}, f_{\mathrm{T}}, \tau$, and $K$ to the barrier shape since different approximations could lead to results qualitatively not concordant. For this reason, the double proton tunnel tautomerization may be considered an interesting subject not concluded yet, and as such it would deserve more attention as a possible source of nonradiative process. Finally, the role of $\mathrm{H}$ bonds in the hypochromism of the double-stranded polynucleotides calls for further studies of model compounds. A study along these lines is underway and will be published at a later time.

Acknowledgment. We are grateful to Mr. Mendoza, director of SPED, for allocation of computer time. J.M. and O.M.S. thank CONICET, CIC, and the Organization of American States for research grants in support of this work.

(52) M. C. Gutzwiller, J. Math. Phys. (N.Y.), 8, 1979 (1967); 10, 1004 (1969); 11, 1791 (1970); 12, 343 (1971).

(53) S. Coleman, "The Uses of Instantons", Lectures given at the 1977 International School of Subnuclear Physics, Ettore Majorana.

(54) A point in Minkowski space is labeled $x^{u}$, where $u=0,1,2,3$, and $x^{0}$ is the time coordinate. As it is well-known, Euclidean space is obtained from Minkowski space by formal analytic continuation in the time coordinate, $x^{4}=-i x^{0}$. A point in Euclidean space is labeled $x^{u}$, where $u=$ $1,2,3,4$.

(55) The Euclidean action is defined as $-i$ times the continuation of the Minkowskian action. Note that $x \cdot y$ in Minkowski space continues to $-x \cdot y$ in Euclidean space.

(56) Shau-Jin Chang, Physica A (Amsterdam), 96, 183 (1979); "Semiclassical Methods in Field Theories", Lectures given at the International Summer Institute on Theoretical Physics, held in Keiserslautern, Germany, Aug 1979. 